\title{
ORIGINAL ARTICLE Oral erlotinib, but not rapamycin, causes modest acceleration of bladder and hindlimb recovery from spinal cord injury in rats
}

\author{
J Kjell, K Pernold, L Olson and MB Abrams
}

Objectives: Erlotinib and Rapamycin are both in clinical use and experimental inhibition of their respective molecular targets, EGFR and mTORC1, has improved recovery from spinal cord injury. Our aim was to determine if daily Erlotinib or Rapamycin treatment started directly after spinal contusion injury in rats improves locomotion function or recovery of bladder function.

Setting: Stockholm, Sweden

Methods: Rats were subjected to contusion injuries and treated during the acute phase with either Erlotinib or Rapamycin. Recovery of bladder function was monitored by measuring residual urine volume and hindlimb locomotion assessed by open-field observations using the BBB rating scale as well as by automated registration of gait parameters. Body weights were monitored. To determine whether Erlotinib and Rapamycin inhibit the same signaling pathway, a cell culture system and western blots were used.

Results: Erlotinib accelerated locomotor recovery and slightly improved bladder recovery; however, we found no long-term improvements of locomotor function. Rapamycin did neither improved locomotor function nor bladder recovery. In vitro studies confirmed that Erlotinib and Rapamycin both inhibit the EGFR-mTORC1 signaling pathway.

Conclusion: We conclude that none of these two drug regimes improved long-term functional outcome in our current model of spinal cord injury. Nevertheless, oral treatment with Erlotinib may offer modest temporary advantages, whereas treatment with Rapamycin does not.

Spinal Cord (2014) 52, 186-190; doi:10.1038/sc.2013.166; published online 21 January 2014

Keywords: erlotinib; rapamycin; locomotion; bladder function

\section{INTRODUCTION}

Several promising experimental treatments for spinal cord injury are based on drugs that have not been tested in humans, making it a demanding task to translate the findings to clinical trials. If drugs that are already in clinical use for other indications could be found to be beneficial in experimental spinal cord injury, the translational process would be both safer and faster. ${ }^{1}$ For example, a diverse set of cancer drugs, targeting more or less specific cellular mechanisms, have been shown to be effective in several other pathologies, including central nervous system disorders. In spinal cord injury, no medical treatment exists that can counteract the disabilities caused by the injury; thus there is a dire need for novel experimental treatments with translational potential. Along these lines, the cancer drug Imatinib (Glivec, Novartis, Horsham, UK) was recently shown to have beneficial effects in experimental spinal cord injury. ${ }^{2}$

Treatment of acute experimental spinal cord injury with a potent epidermal growth factor receptor (EGFR) inhibitor (PD168393) has been shown to improve functional outcome substantially through rescue of spinal tissue. ${ }^{3}$ Although PD168393 is not in clinical use, the results suggest that clinically used EGFR inhibitors may have similar effects. This renders Erlotinib, an EGFR inhibitor used to treat certain forms of cancer, an interesting candidate drug, which, if positive effects were to be found in animals, would allow faster translation to clinical trials.
The mammalian target of rapamycin complex 1 (mTORC1) pathway constitutes a possible mediator of the improved outcome after EGFR inhibition in experimental spinal cord injury. This is supported by our preliminary findings that activation of mTORC1 is prominent in immune cells and reactive astrocytes after spinal cord injury (Kjell 2014, under review). Rapamycin, which inhibits mTORC1, is currently used to treat dermal Kaposi's sarcoma and also as an immunosuppressant in organ transplantations. The drug also has been reported to increase autophagy in neurons and to improve functional recovery after administration during the acute phase in experimental spinal cord injury in mice. ${ }^{4}$

On the basis of the above reasoning, the purpose of the present study was to test if two drugs used in the clinic for other indications might also have beneficial effects in acute spinal cord injury. We used a well-characterized model of experimental spinal cord injury in rats to test if EGFR inhibition with Erlotinib or downstream inhibition of EGFR signaling with Rapamycin (Figure 1) might have effects akin to those previously observed by treatment with PD168393 or Glivec.

\section{MATERIALS AND METHODS}

Spinal cord injury

Animal work was approved by the Northern Stockholm Ethical Committee and in accordance with the Helsinki declaration. Female 200-225 g SpragueDawley rats (Scanbur, Germany) were subjected to a mild $(n=27)$ or 


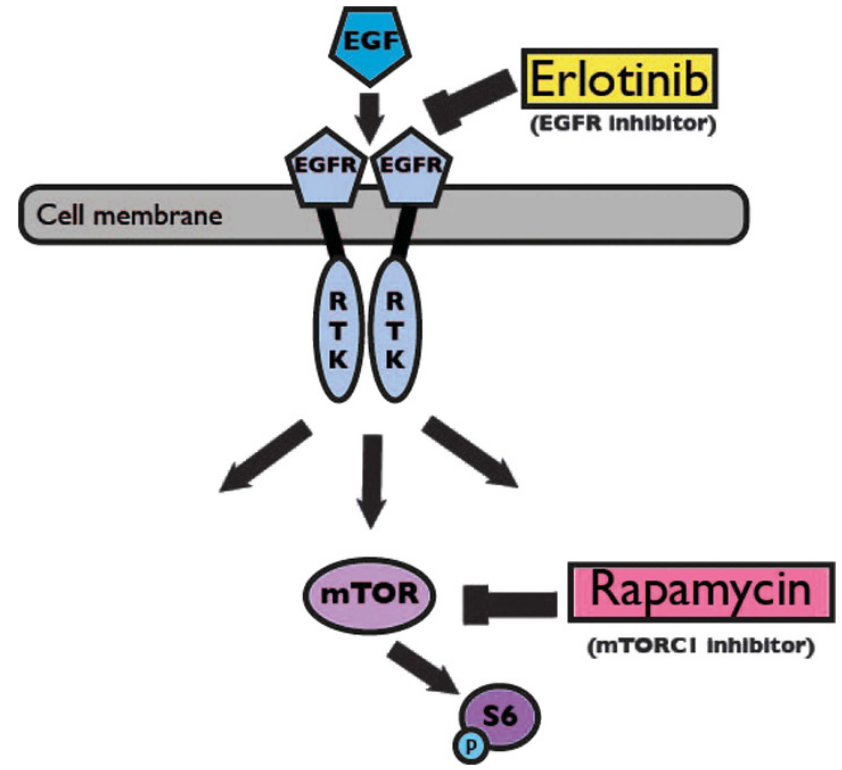

Figure 1 The EGFR-mTOR pathway. Schematic figure displaying the molecular targets of Erlotinib and Rapamycin. EGFR, epidermal growth factor receptor; RTK, receptor tyrosine kinase; mTORC1, mammalian target of rapamycin complex 1; S6, ribosomal protein $\mathrm{S} 6$.

moderate $(n=16)$ spinal contusion injury by weight-drop, as previously described. ${ }^{5,6}$ Postoperative care included 3 days of analgesics (bruprenorphine $0.015 \mathrm{mg} \mathrm{kg}^{-1}$, Temgesic, i.p.), 7 days of prophylactic antibiotics $\left(0.6 \mathrm{mg} \mathrm{kg}^{-1}\right.$ trimethoprim, Borgal, Hoechst, AG), and manual bladder emptying twice daily until animals regained bladder function. Bladder emptying was carried out by applying pressure to the lowermost central part of the belly using the index and middle finger. As urine is expelled, it is collected in a tube and the volume of urine is registered. Animals were housed $3 /$ cage at $24-26^{\circ} \mathrm{C}$ with a $12 / 12 \mathrm{~h}$ light/dark cycle and water and food ad libitum.

\section{Drugs and treatments}

Rats exposed to mild injury were randomized into three groups ( $n=9 /$ group) and given PBS (Gibco, Paisley, UK), Erlotinib $\left(5 \mathrm{mg} \mathrm{kg}^{-1}\right.$, Tarceva, Roche, Welmyn Garden City, UK) or Rapamycin $\left(1.5 \mathrm{mg} \mathrm{kg}^{-1}\right.$, Sirolimus, Wyeth, Berkshire, UK) starting $30 \mathrm{~min}$ after injury. Erlotinib treatment was then administered once daily until 5 days post injury, whereas Rapamycin was administered once daily until 14 days post injury. One animal each from the PBS and Rapamycin group was removed from final analysis due to being insufficiently injured, determined by no loss of bladder function and close to full locomotor function. Rats exposed to moderate injury were randomized into two groups ( $n=8 /$ group) and given PBS or Rapamycin starting $30 \mathrm{~min}$ after injury. Tarceva pills with Erlotinib as active substance, were grinded and mixed with PBS. Rapamycin was available as a pre-mixed fluid (Rapamune). All treatments were given orally by gavage. Epidermal growth factor (EGF) (Sigma) was used to stimulate spinal astrocytes in culture and Erlotinib (LC laboratories, Woburn, MA, USA) was used to inhibit EGFR activation in such cultures.

\section{Open-field locomotion test}

Assessment of hindlimb function in an open field was assessed using the BBB locomotor rating scale and $\mathrm{BBB}$ subscore as described previously. ${ }^{7,8}$ The BBB subscore records paw position, toe clearance, trunk stability and tail position. Scores were made weekly during 10 weeks (except at week 5), by two experimenters blinded to treatment groups. The scores from the two experimenters were averaged for each individual animal prior to statistical analysis. During the first 2 weeks injured rats may experience spasticity that causes contraction of one or both hindlimbs. After mild injury, three rats at week 1 and two rats at week 2 had a contracted leg during the 4-min assessment period in the Rapamycin group. We assigned these rats a BBB score based only on the non-contracted leg, resulting in the data presented. If the score for the contracted leg (zero) had been considered, the average score would have been lower than presented for both weeks and significantly lower compared with the PBS group at week 1 .

\section{Quantitative locomotion assessment}

Automated assessment of locomotion and walking parameters (Catwalk, Noldus, Wageningen, Netherlands) was obtained as previously described. ${ }^{9}$ Pre-injury measurements were used as baseline values and all post-injury measurements were normalized to the baseline values. A regularity index was obtained to reflect to what degree the rats were able to run according to defined patterns of orderly limb use.

\section{Cell culture}

Spinal astrocyte cultures were prepared from rats $(180 \mathrm{~g})$ as described previously. ${ }^{10}$ Prior to drug challenge, cell preparations $(n=4)$ were starved for $48 \mathrm{~h}$ and then stimulated by EGF ( $100 \mathrm{ng} \mathrm{ml}^{-1}$, Sigma, St Louis, MO, USA) dissolved in dimethylsulfoxide (Sigma). Fifteen minutes later cell lysates were collected. Erlotinib (1 um, LC laboratories) was used for EGFR inhibition and added to the medium $30 \mathrm{~min}$ prior to EGF stimulation.

\section{Western blot}

Spinal astrocytes were homogenized and collected in RIPA lysis buffer with added protease and a cocktail of phosphatase inhibitors (Roche, Mannheim, Germany), sonicated and centrifuged after which the supernatant was collected. Protein concentration was measured using a BCA-assay to determine gel-loading dilution. Samples loaded into a 4-12\% Bis-Tris gel (Invitrogen, Carlsbad, CA, USA) were pre-boiled for $3 \mathrm{~min}$ and contained 10\% DTT, and all samples were diluted to the same concentration using LB xl. MOPs buffer (NuPage, Invitrogen) was used as running buffer and samples ran until reaching end of gel (Novex western blot system, Invitrogen). Tranfers were done onto a PVDF membrane (Millipore, Temecula, CA, USA) using an added transfer buffer mix (NuPage). For western blot staining, membranes were preblocked in 50\% TBS and 50\% blocking buffer (Licor, Lincoln, NE, USA) and then incubated with primary antibodies pS6 and S6 (Cell signaling, Danvers, MA, USA) in $50 \%$ TBS- $0.1 \%$ tween and $50 \%$ blocking buffer. After washing in TBS- $0.1 \%$ tween, secondary antibodies (Odessey IRDye 680 and 800, Licor) were added and membranes incubated for $1 \mathrm{~h}$. Membranes were next washed with TBS- $0.1 \%$ tween and lastly once with TBS before being scanned (LICOR IR scanner). An appropriate program (Odyssey scanner) was used for quantification of band intensity.

\section{Statistics}

One-way ANOVAs with post hoc Bonferroni tests were used to analyze western blot data. Two-way ANOVAs with post-hoc Bonferroni tests were used to analyze BBB scores, residual urine and Catwalk data. Subscores were analyzed using a non-parametric Mann-Whitney $U$-test.

\section{Statement of ethics}

We certify that all applicable institutional and governmental regulations concerning the ethical use of animals were followed during the course of this research.

\section{RESULTS}

\section{Erlotinib and Rapamycin: mild injury}

The effects of Erlotinib and Rapamycin treatment after mild contusion injury was assessed during 10 weeks by BBB scoring, automated locomotion assessment and residual urine measurements. Open-field locomotion assessment revealed an initial difference in the BBB score for Erlotinib, but not for Rapamycin treated animals compared with controls (Figure 2a). The Erlotinib group had significantly higher average BBB scores 1 and 2 weeks post injury, $9.8 \pm 0.6$ and $12.9 \pm 0.1$, 

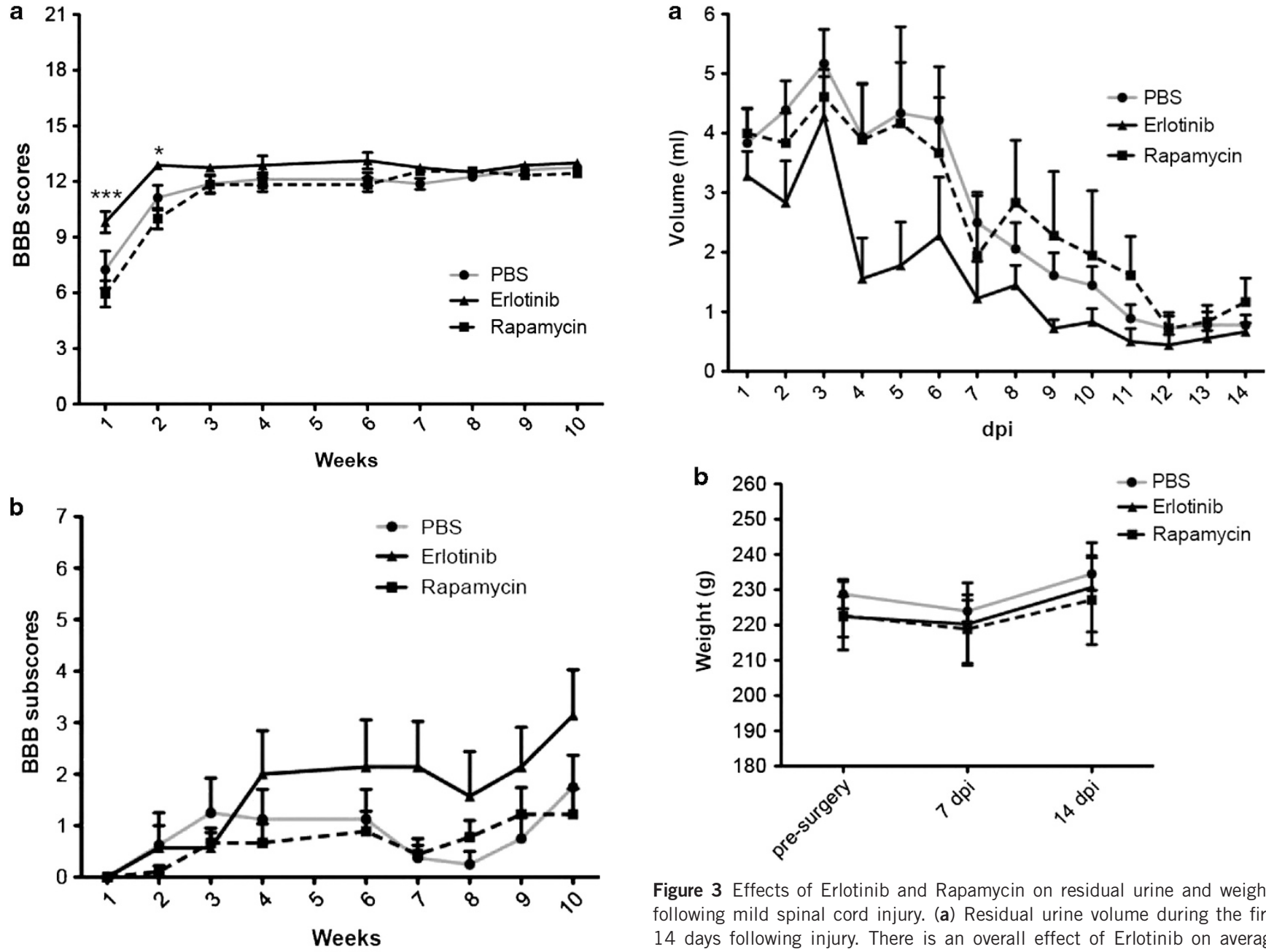

Figure 3 Effects of Erlotinib and Rapamycin on residual urine and weights following mild spinal cord injury. (a) Residual urine volume during the first 14 days following injury. There is an overall effect of Erlotinib on average residual urine during this period $(P<0.001)$. (b) Body weights before and 1 and 2 weeks after injury.

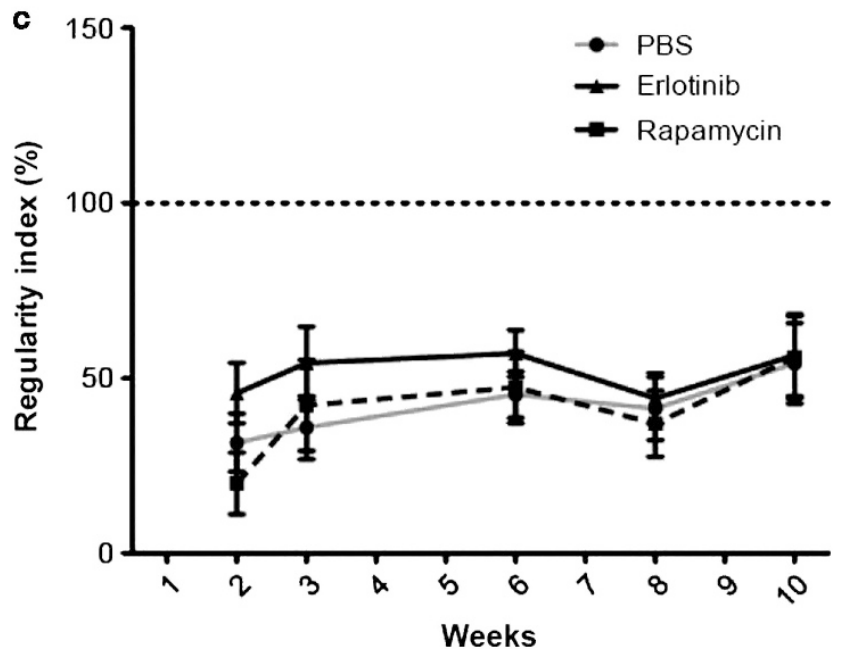

Figure 2 Effects of Erlotinib and Rapamycin on BBB scores, BBB subscores and regularity index following mild spinal cord injury. (a) Locomotion assessed in an open field using the BBB score. In addition to the significantly better scores in the Erlotinib group at weeks 1 and 2, there is an overall effect of Erlotinib on BBB scores $(P<0.001)$ compared with the control group. (b) BBB subscore. (c) Regularity index, a measure of coordination, as recorded by the Catwalk device. Data presented as mean \pm s.e.m. ${ }^{*} P<0.05,{ }^{* * *} P<0.001$

compared with the control group, $7.3 \pm 1.0$ and 11.1 \pm .0 .7 , respectively. Rapamycin, treatment, however, did not cause any significant alteration of BBB scores. At the last time point of the experiment, 10 weeks after injury, the Erlotinib, Rapamycin and control groups had BBB scores of $13 \pm 0.2,12.4 \pm 0.2$ and $12.8 \pm 0.2$, respectively. Nevertheless, we found an overall treatment effect of Erlotinib compared with PBS $(P<0.001)$. The BBB subscores were not significantly different between groups at any individual time point. The 10 weeks post-injury scores for Erlotinib, Rapamycin and the control group were $3.5 \pm 2.4,1.2 \pm 1.5$ and $1.8 \pm 1.8$, respectively (Figure 2b). Likewise, other automated measurements such as base of support, swing time and stance time did not display any significant differences between groups at the final time point, 10 weeks after injury (data not shown).

Residual urine measurements were carried out as an indicator of bladder function each day for 3 weeks (Figure 3a). Average residual urine volumes tended to be lower in the Erlotinib group than in the other two groups $(P<0.001)$. The control group and the Rapamycin group had similar recovery.

Weight was recorded before the surgical procedure and 1 and 2 weeks later (Figure 3b). No significant differences of body weights between treatment groups were noted during the first 2 weeks after mild spinal cord injury. 
Rapamycin: moderate injury

Possible effects of Rapamycin treatment were also assessed after a moderate contusion injury using $\mathrm{BBB}$ scoring and residual urine measurements. These animals were followed for 17 weeks post injury. There was a tendency for an overall treatment effect $(P=0.815)$, albeit modest in magnitude such that none of the individual weekly scores were significantly different between groups. At 17 weeks after injury, the scores were $8.3 \pm 0.2$ and $8.5 \pm 0.2$ for the control and Rapamycin group, respectively (Figure 4a). Likewise, residual urine measurements during the first 4 weeks after injury did not indicate any improved recovery of bladder function caused by treatment
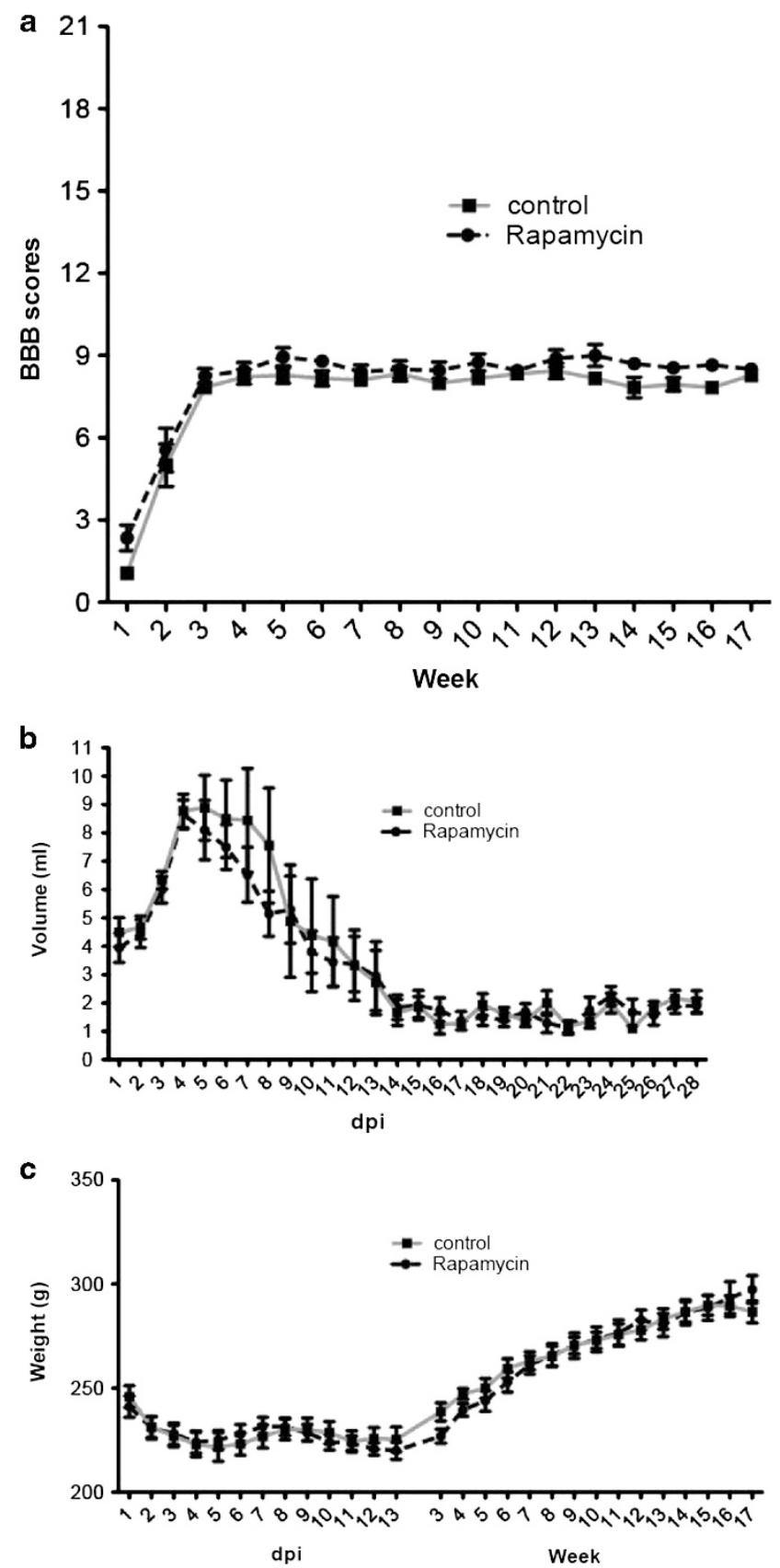

Figure 4 Effects of Rapamycin on BBB scores, residual urine and weights following moderate spinal cord injury. (a) BBB scores. (b) Residual urine volume. (c) Body weights. Treated animals received Rapamycin daily for the first 14 days.
(Figure 4b). Weights, recorded daily during the first 13 days after injury and weekly thereafter, were similar in control and Rapamycintreated animals (Figure 4c).

\section{In vitro: EGFR-mTOR pathway}

Spinal astrocytes were cultured and when confluent starved $48 \mathrm{~h}$ prior to stimulation. Phosphorylation of the S6 protein was used as a readout for mTORC1 activation, as S6 is downstream of mTORC1 (Figure 1). All measurements were normalized to non-stimulated cells. As follows from Figure 5, EGF stimulation resulted in a robust $1.97 \pm 0.12$ fold increase of S6 phosphorylation compared with a $1.12 \pm 0.27$ fold change for controls treated with vehicle only (dimethylsulfoxide). Erlotinib, the active ingredient in Tarceva, almost completely blocked the EGF-induced increase of S6 phosphorylation (fold change: $1.28 \pm 0.22$ ).

\section{DISCUSSION}

The aim of this study was to determine whether Erlotinib would improve functional outcome and if Rapamycin would have similar effects as mTORC1 is downstream of EGFR (Figure 1). We found that Erlotinib significantly improved BBB scores in the first 2 weeks, thus indicating accelerated recovery. We also found decreased residual urine with Erlotinib treatment. Rapamycin treatment had no significant effects on functional recovery from mild or moderate spinal cord injury in our model system.

There is currently no drug in clinical use for treatment of spinal cord injury. The findings that the EGFR inhibitor PD168393 has robust positive effects on recovery from spinal cord injury in rats, ${ }^{3}$ that oral Imatinib also has robust effects, ${ }^{2}$ and that Erlotinib has modest but significant effects as shown in the current study, together indicate that targeting receptor tyrosine kinases in the acute phase of spinal cord injury constitutes one interesting way to treat spinal cord injury, an approach which could also be combined with other treatments.

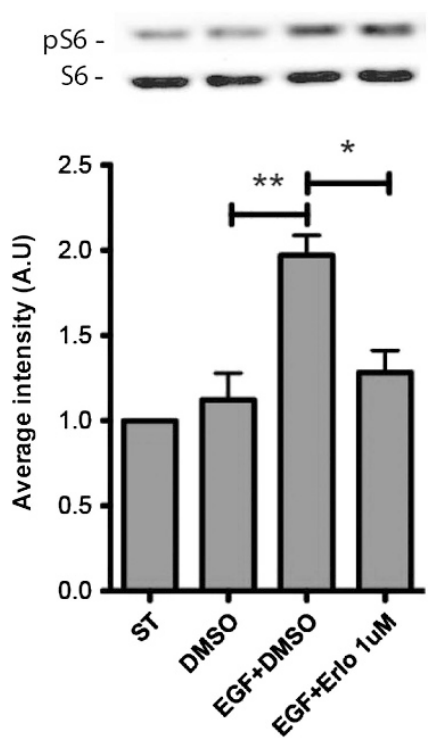

Figure 5 Erlotinib blocks EGF activation of the mTOR pathway. Spinal cordderived astrocyte cultures were used to monitor the ability of Erlotinib to reduce S6 phosphorylation after EGF stimulation. Top: representative image of a western blot. Bottom: quantification of western blot results. Data presented as mean \pm s.e.m. ${ }^{*} P<0.05,{ }^{*} P<0.01$. 
In this study, systemic administration of the reversible EGFR inhibitor Erlotinib did not improve recovery in a lasting manner as seen after local delivery of the strong irreversible EGFR inhibitor PD168393. ${ }^{3}$ Both PD168393 and Erlotinib may pass the compromised blood-brain-barrier after injury, but delivery of PD168393 directly to the site of injury during 2 weeks is likely to lead to higher concentration of active substance compared with oral administration of Erlotinib. ${ }^{3,11,12}$ Recommended doses of cancer drugs, however, are typically based on toxicity, rather then efficacy. Erlotinib toxicity is very similar in rats and humans, which should help facilitate a possible translational process. ${ }^{13}$ Thus, Erlotinib was given to rats in a clinically relevant dose equivalent to a dose that could be tolerated without major adverse events (EMA Tarceva report) if administered to humans for several consecutive days, with bioavailability taken into account. ${ }^{14}$ Higher doses would not be recommended unless combined with a vehicle that reduces toxicity, such as nanoencapsulation. ${ }^{15}$ In summary, the local administration, the difference in length of the administration period, the difference in potency, and the difference in concentration may all explain the increased efficacy of PD168393 as compared with that of Erlotinib. Our data do not exclude the possibility that Erlotinib might be beneficial also clinically, particularly in combination with other drugs. In humans, drugassisted accelerated recovery, even if only to the same final functional state as without treatment, might not be without advantages, such as helping to avoid urinary infections and other dysfunctions associated with spinal cord injury.

Complex 1 of mTOR is downstream of EGFR and acute systemic mTORC1 inhibition with Rapamycin after spinal cord injury in mice has recently been reported to have positive effects on functional recovery; however, we found no robust functional improvements after treating a mild or moderate contusion injury in rats with Rapamycin. Thus these results differ from previous results in mice, in which Rapamycin was administered once i.p. $4 \mathrm{~h}$ after injury at a dose of $1 \mathrm{mg} \mathrm{kg}^{-1}$. In our experiments, the first $1.5 \mathrm{mg} \mathrm{kg}^{-1}$ dose was administered orally by gavage $30 \mathrm{~min}$ after injury and was followed by once daily doses for 14 days. To intervene in the acute phase after injury by inhibition of mTORC1 activation seems reasonable, as mTORC1 is highly activated in immune cells maintaining pro-inflammatory activity (Kjell 2014, under review). It should be noted that mice and rats undergo different inflammatory reactions after injury, resulting in somewhat different pathology. ${ }^{16}$ mTOR signaling is complex, and differences in drug administration or between species may thus lead to different outcomes. mTOR is also reportedly involved in pain mechanisms, ${ }^{17}$ and it remains to be investigated whether Rapamycin treatment has any effect on pain or sensory function after spinal cord injury.

We recently found that another receptor tyrosine kinase inhibitor and cancer drug, Imatinib, has robust positive effects on recovery from experimental spinal cord injury when administered in the same manner as Erlotinib was delivered in the present study. ${ }^{2}$ Together, the findings with PD168393, Imatinib, and the significant although modest effects of Erlotinib noted here, suggests that receptor tyrosine kinase inhibition is one way in which some of the secondary negative effects of spinal cord injury may be counteracted, leading to better recovery. Further work is needed to determine if Erlotinib or Rapamycin may be of interest in the development of treatment strategies for spinal cord injury.

\section{DATA ARCHIVING}

There were no data to deposit.

\section{CONFLICT OF INTEREST}

The authors declare no conflict of interest.

\section{ACKNOWLEDGEMENTS}

We thank professor Anna Josephson for constructive review of the manuscript. Supported by Vinnova, Wings for Life, the Swedish Research Council, The Karolinska DPA award, Karolinska institutet.

1 Ashburn TT, Thor KB. Drug repositioning: identifying and developing new uses for existing drugs. Nat Rev Drug Discov 2004; 3: 673-683.

2 Abrams MB, Nilsson I, Lewandowski SA, Kjell J, Codeluppi S, Olson L et al. Imatinib enhances functional outcome after spinal cord injury. PLoS One Public Lib Sci 2012; 7: e38760

3 Erschbamer M, Pernold K, Olson L. Inhibiting epidermal growth factor receptor improves structural, locomotor, sensory, and bladder recovery from experimental spinal cord injury. J Neurosci 2007; 27: 6428-6435.

4 Sekiguchi A, Kanno H, Ozawa H, Yamaya S, Itoi E. Rapamycin promotes autophagy and reduces neural tissue damage and locomotor impairment after spinal cord injury in mice. J Neurotrauma 2011; 29: 946-956.

5 Gruner JA. A Monitored contusion model of spinal cord injury in the rat. J Neurotrauma 1992; 9: 123-128.

6 Kjell J, Josephson A, Abrams MB, Sandor K, Svensson Cl. Rat substrains differ in the magnitude of spontaneous locomotor recovery and in the development of mechanical hypersensitivity after experimental spinal cord injury. J Neurotrauma 2013; 30: 1805-1811.

7 Basso DM, Fisher LC, Anderson AJ, Jakeman LB, McTigue DM, Popovich PG. Basso Mouse Scale for locomotion detects differences in recovery after spinal cord injury in five common mouse strains. J Neurotrauma 2006; 23: 635-659.

8 Lankhorst AJ, Verzijl MR, Hamers FPT. Experimental spinal cord contusion injury: comparison of different outcome parameters. Neurosci Res Commun John Wiley \& Sons, Inc 1999; 24: 135-148.

9 Hamers FP, Lankhorst AJ, van Laar TJ, Veldhuis WB, Gispen WH. Automated quantitative gait analysis during overground locomotion in the rat: its application to spinal cord contusion and transection injuries. J Neurotrauma 2001; 18 187-201.

10 Codeluppi S, Gregory EN, Kjell J, Wigerblad G, Olson L, Svensson Cl. Influence of rat substrain and growth conditions on the characteristics of primary cultures of adult rat spinal cord astrocytes. J Neurosci Methods 2011; 197: 118-127.

11 Pardridge WM. Molecular biology of the blood-brain barrier. Mol Biotechnol 2005; 30 : 57-70.

12 Habgood MD, Bye N, Dziegielewska KM, Ek CJ, Lane MA, Potter A et al. Changes in blood-brain barrier permeability to large and small molecules following traumatic brain injury in mice. Eur J Neurosci 2007; 25: 231-238.

13 Hoshino-Yoshino A, Kato M, Nakano K, Ishigai M, Kudo T, Ito K. Bridging from preclinical to clinical studies for tyrosine kinase inhibitors based on pharmacokinetics/ pharmacodynamics and toxicokinetics/toxicodynamics. Drug Metab Pharmacokinet 2011: 26: 612-620.

14 Frohna P, Lu J, Eppler S, Hamilton M, Wolf J, Rakhit A et al. Evaluation of the absolute oral bioavailability and bioequivalence of erlotinib, an inhibitor of the epidermal growth factor receptor tyrosine kinase, in a randomized, crossover study in healthy subjects. J Clin Pharmacol 2006; 46: 282-290.

15 Marslin G, Sheeba CJ, Kalaichelvan VK, Manavalan R, Neelakanta Reddy P, Franklin G. Poly(D,L-lactic-co-glycolic acid) nanoencapsulation reduces Erlotinib-induced subacute toxicity in rat. j biomed nanotechnol. American Scientific Publishers 2009; 5: 464-471.

16 Sroga JM, Jones TB, Kigerl KA, McGaughy VM, Popovich PG. Rats and mice exhibit distinct inflammatory reactions after spinal cord injury. J Comp Neurol 2003; 462. 223-240.

17 Géranton SM, Jiménez-Díaz L, Torsney C, Tochiki KK, Stuart SA, Leith JL et al. A rapamycin-sensitive signaling pathway is essential for the full expression of persistent pain states. J Neurosci 2009; 29: 15017-15027.

(c) (i)(2) This work is licensed under a Creative Commons Attribution-NonCommercial-ShareAlike 3.0 Unported License. To view a copy of this license, visit http://creativecommons. org/licenses/by-nc-sa/3.0/ 